\title{
Salir adelante: subjetividades políticas en primera infancia y familias en el destierro
}

\author{
María Camila Ospina-Alvarado, Ph. D. ${ }^{a}$ \\ Centro de Estudios Avanzados en Niñez y Juventud, \\ Cinde-Universidad de Manizales, Colombia \\ María Teresa Luna, Ph. D. ${ }^{b}$ \\ Cinde-Universidad de Manizales, Colombia \\ Sara Victoria Alvarado, Ph. D. ${ }^{c}$ \\ Centro de Estudios Avanzados en Niñez y Juventud, \\ Cinde-Universidad de Manizales, Colombia
}

\section{Resumen (analítico)}

Se presentan resultados de una investigación que problematiza el proceso de construcción de subjetividades políticas de niños y niñas de primera infancia cuyas familias han vivido el conflicto armado colombiano. Epistémica y metodológicamente se empleó la hermenéutica ontológica política, enfatizando las narrativas colectivas y generativas de 19 niñas, 25 niños, 23 madres, 7 padres, 15 maestras y 1 maestro, en dos Centros de Desarrollo Infantil en Bogotá y Pereira. Se encontró que salir adelante — como metáfora que da cuenta de las subjetividades de los niños y las niñas-involucra una opción por las relaciones, romper la homogeneización, prácticas de resistencia y re-existencia y constitución de subjetividades e identidades políticas. Se concluye que los niños y las niñas tienen disposiciones relacionales como sujetos políticos desde sus primeros años.

\section{Palabras clave}

Primera infancia, familia, conflicto armado, capacidad, identidad.

\section{Thesauro}

Tesauro de Ciencias Sociales de la Unesco.

\section{Para citar este artículo}

Ospina-Alvarado, M. C., Luna, M. T., \& Alvarado, S. V. (2022). Salir adelante: subjetividades políticas en primera infancia y familias en el destierro. Revista Latinoamericana de Ciencias Sociales, Niñez y Juventud, 20(1), 1-25.

https://dx.doi.org/10.11600/rlcsnj.20.1.5012

\section{Historial}

Recibido: 21.09 .2020

Aceptado: 29.04.2021

Publicado: 27.10 .2021

\section{Información artículo}

Este artículo de investigación cualitativa, recoge resultados de la investigación Salir adelante: construcción relacional de subjetividades políticas de niños y niñas de la primera infancia cuyas familias provienen de contextos de conflicto armado, realizada entre agosto del 2016 y julio del 2020. Área: ciencias sociales. Subárea: otras ciencias sociales, interdisciplinaria. 


\section{Moving forward: Political subjectivities in early childhood and families in exile}

\section{Abstract (analytical)}

The article presents the results of a research study that aimed to comprehend and strengthen the political subjectivities of children in early childhood whose families come from the context of armed conflict. Epistemically and methodologically, the research used political and performative ontological hermeneutics. It had an emphasis on the collective and generative narratives of 19 girls, 25 boys, 23 mothers, 7 fathers, 15 female teacher and 1 male teacher in two child care centers in Bogotá and Pereira. The results showed that "moving forward", as a metaphor that refers to children's subjectivities, involves an option for relationships, eliminates homogenization, and prompts resistance and reexistence practices, as well as the constitution of political subjectivities and identities. Participants concluded that the children's lives deserve to be lived because of their condition of humanity.

Keywords

Early childhood, family, armed conflict, potential, identity.

\section{Seguindo em frente: subjetividades políticas na primeira infância e famílias em situação de desterro}

\section{Resumo (analítico)}

São apresentados resultados de uma investigação orientada para entender e fortalecer subjetividades políticas em meninos e meninas da primeira infância cujas famílias são originárias do conflito armado. Utilizou-se a hermenêutica ontológica política ou performativa, epistemológica e metodológica, com ênfase nas narrativas coletivas e generativas de 19 meninas, 25 meninos, 23 mães, 7 pais, 15 professores e uma professora, em dois centros de desenvolvimento infantil em Bogotá e Pereira. Verificou-se que avançar como uma metáfora responsável pelas subjetividades de meninos e meninas envolve uma opção de relacionamento, rompendo com a homogeneização, práticas de resistência e reexistência e constituição de subjetividades e identidades políticas. Conclui-se que a vida de meninos e meninas merece ser vivida em virtude de sua condição humana.

Palavras-chave

Primeira infância, família, conflito armado, capacidade, identidade.

\section{Información autoras}

[a] Psicóloga, Universidad de los Andes. Magíster en Psicología Clínica, Pontificia Universidad Javeriana. Doctora en Estudios de Medios y Comunicación, Universidad Libre de Bruselas-Taos Institute. Doctora en Ciencias Sociales, Niñez y Juventud, Universidad de Manizales-Cinde. H5: 17. Correo electrónico: mospina@cinde.org.co (iD) 0000-0002-7271-151X.

[b] Fonoaudióloga, Universidad Católica de Manizales. Magíster en Desarrollo Educativo y Social, Nova University-Cinde. Doctora en Ciencias Sociales, Niñez y Juventud, Universidad de Manizales-Cinde. H5: 5. Correo electrónico: mtluna@cinde.org.co iD 0000-0002-3967-5844.

[c] Psicóloga, Pontificia Universidad Javeriana. Magíster en Desarrollo Educativo y Social, Nova UniversityCinde. Doctora en Educación, Nova University-Cinde. Posdoctora en Ciencias Sociales, Niñez y Juventud. H5: 21. Correo electrónico: salvarado@cinde.org.co (iD) 0000-0002-0115-8075. 


\section{Introducción}

omo indica el Informe de la Comisión Histórica del Conflicto y sus Víctimas (2015),
el conflicto armado colombiano ha tenido implicaciones tanto estructurales como subjetivas en su consolidación y mantenimiento; es uno de los conflictos con mayor complejidad en cuanto a sus actores, sectores e intereses involucrados, que han intervenido en su duración y alcance, con expresiones diferenciales en los territorios y en las comunidades. Sus implicaciones estructurales y subjetivas han sido aceptadas ampliamente en diversos estudios y recuentos históricos (Alvarado et al., 2012; Castellanos \& Torres, 2008; Contreras, 2003; González et al., 2002). Por su parte, Uribe (1999) señala que la emergencia y la continuidad del conflicto tiene que ver con la precariedad ${ }^{1}$ presente en los territorios colombianos, así como con la debilidad institucional, en lo que ha denominado la «soberanía en vilo»:

El estado de guerra cuasipermanente y su correlato, la soberanía en vilo, se expresan también en la debilidad del referente institucional, un tema reiterativo en los estudios sobre la violencia en Colombia y que ha sido visto generalmente como ausencia de Estado (...). El orden institucional público, aunque pudiera mostrar omnipresencia en la nación no ha tenido omnipotencia, no constituye una autoridad razonablemente acatada o violentamente impuesta, pese a los esfuerzos del gobierno en un sentido y en otro. Sin omnipotencia institucional, es decir, sin una autoridad única, suprema y universal dentro del territorio, no hay soberanía en sentido absoluto. Además, el orden institucional público presenta serios problemas en la esfera de la soberanía representada. (p. 3o)

Si bien la ausencia estatal es una de las conclusiones más aceptadas cuando se interroga acerca del rol del Estado en la continuidad del conflicto armado en Colombia, otros estudios (como el de Garay et al., 2008) revelan que, más que ausencia, se trata de una cooptación por intereses que favorece el mantenimiento de la estructura hegemónica y

\footnotetext{
${ }^{1}$ Precariedad que se expresa en el hecho de que Colombia se ubica en el cuarto puesto de inequidad a nivel mundial (Banco Mundial, 2019).
} 
tradicional de los poderes, como sucede con las fuerzas paramilitares o de derecha extrema armada que cuentan con la connivencia por parte del Estado.

En dicho contexto, paradójicamente, mientras el Estado tendría que ser el garante de los derechos humanos y del bienestar de las comunidades, en particular de los niños y las niñas, ellos y ellas han sido olvidados (como se identifica en la revisión de informes y estudios previos; Alvarado et al., 2012), posicionados como víctimas (Niño, 2012) o como parte activa y colaboradores de los actores armados (Coalico, 2019; Sierra et al., 2009), así como han experimentado la naturalización de la violencia como parte cotidiana de la socialización en sus familias y comunidades (Ceballos \& Bello, 2001; Defensoría del Pueblo, 2002; Lugo, 2017; Niño, 2012; Torrado et al., 2002; Torrado et al., 2009; Villanueva O'Driscoll, 2013; Villanueva et al., 2017).

Esta situación se ha replicado en otros conflictos armados y guerras en el mundo. Los estudios previos al respecto, consultados en las bases de datos Embase y Pubmed, ${ }^{2}$ dan cuenta de la vulneración de los derechos de los niños y las niñas (Boothby et al., 20o6; Bradley, 2018; Catani et al., 2010; Cummings et al., 2017; Feldman et al., 2010; Jordans et al., 2016; Peltonen \& Punamäki, 2010; Richter et al., 2018; Shenoda et al., 2018; Slone \& Mann, 2016; Sommer et al., 2018; Yurtbay et al., 2003), así como de la afectación de la primera infancia a causa de la violencia vivida por sus familias (Andersson, 2015; Bradley, 2018; Catani et al., 2010; Cummings et al., 2017; Demause, 2008; Feldman et al., 2010; Fossion et al., 2013; Jordans et al., 2016; Llabre \& Hadi, 2009; Llabre et al., 2015; Nagata, 1991; Panter-Brick et al., 2015; Peltonen \& Punamäki, 2010; Pye \& Simpson, 2017; Richter et al., 2018; Shenoda et al., 2018; Slone \& Mann, 2016; Sommer et al., 2018; Yurtbay et al., 2003).

Solo algunos estudios de los referidos en los antecedentes de la presente investigación centran su atención en la primera infancia en contextos de conflicto armado en Colombia (Ila et al., 2009; Romero \& Castañeda, 2009; Torrado et al., 2009). Sin embargo, diversos autores mencionan que la mayor vulneración de los derechos, así como los mayores aprendizajes, se dan en los primeros años de vida (Alvarado et al., 2012; Ceballos \& Bello, 2001; Human Rights Watch, 2003; Sierra et al., 2009; Torrado et al., 2002). Cabe señalar que algunos de estos estudios han retomado los relatos de los propios niños y niñas o de sus agentes relacionales. Por su parte, las cifras también dan cuenta de la afectación en esta etapa del ciclo vital: a febrero del 2020 el número de niños y niñas de o a 5 años víctimas

\footnotetext{
${ }^{2}$ Revisión que, entre el 2000 y el 2019, arrojó veintiocho estudios con los siguientes términos de búsqueda: conflictos armados, guerra y psicología en relación con niños y niñas de la primera infancia, menores de seis años, y niños y niñas entre los seis y los doce años (Ospina-Alvarado, 202ob, p. 24 ).
} 
del conflicto armado en nuestro país ascendía a 335 999, según el Registro Único de Víctimas (Unidad para la Atención y Reparación Integral a las Víctimas, 2020). La mayor afectación vivenciada por los niños y las niñas en dichas edades se debe al desplazamiento forzado de sus familias, presente en el 96.33\% de los casos según datos de 2018 de la Red Nacional de Información (Save the Children, 2018). El desplazamiento forzado no ha sido vivido únicamente como una pérdida material sino también simbólica, entendida como destierro, similar a lo referido por Gaviria (2012), y ha aportado en la configuración de los propios niños y niñas y de las prácticas relacionales en las que participan.

En este sentido, y teniendo en cuenta la afectación vivida durante los primeros años de vida en los contextos de conflicto armado referida en estudios previos, el gran potencial presente en dicho momento del ciclo vital y la relevancia de las relaciones y de las conversaciones establecidas en la construcción social de las subjetividades, la investigación a la base del presente artículo buscó comprender los modos en que los niños y las niñas de primera infancia, cuyas familias han vivido el contexto de conflicto armado colombiano, construyen sus subjetividades a partir de procesos dialógicos y de reconstrucción de memorias en las relaciones en las que participan, así como aportar al posicionamiento de estos niños y niñas como sujetos políticos para la paz. Este artículo se enfoca en dos de las preguntas del estudio: ¿cómo es el proceso de construcción relacional y social de subjetividades de niños y niñas de la primera infancia en contextos de conflicto armado?; ¿cómo se consolidan los procesos de socialización política y de construcción de subjetividades políticas en el salir adelante como práctica de reconciliación de las familias y de agenciamientos compartidos de los niños y las niñas de la primera infancia?

La relevancia de la comprensión de la construcción relacional y social de las subjetividades de los niños y las niñas radica en la posibilidad de deconstruir los relatos hegemónicos de violencia y vulneración, así como de coconstruir relatos alternativos y prácticas relacionales mediante las cuales ellos, ellas y sus familias se posicionen activamente como sujetos políticos y se alejen de la reproducción de las violencias vividas en los contextos de conflicto armado.

El presente artículo hace énfasis en resultados de investigación que articulan las voces de niños y niñas de la primera infancia, de sus familias provenientes de contextos de conflicto armado y de docentes. Para esto se toman referentes conceptuales del construccionismo social (Anderson, 2012; Davies \& Harré, 1999; Gergen, 1996, 2006, 2007, 2009, 2012; Gergen \& Gergen, 2012; McNamee, 2015; Ospina-Alvarado, 2018, 2020a; Shotter, 2008), como vía para comprender la construcción relacional y social de sus subjetividades, la 
terapia narrativa (White \& Epston, 1993), como posibilitadora de narrativas alternativas a la de la violencia alrededor de sus historias de vida, y la socialización política (Alvarado et al., 2012; Alvarado \& Ospina-Alvarado, 2009; Luna, 2018), como horizonte que facilita el agenciamiento de los niños y las niñas desde sus primeros años de vida, en diálogo con otras aproximaciones críticas (Agamben, 1998; Arendt, 2009; Camps, 2011; Levinas, 2006; Mèlich, 200o; Pedraza, 1999; Santos, 2011; Segato, 2016; Sosenski \& Jackson, 2012) y con algunos pensadores clásicos (Derrida, 1998; Foucault, 2009; Gadamer, 1993; Heidegger, 1951), como se verá en los resultados y la discusión.

\section{Método}

El abordaje epistémico y metodológico de la investigación fue el de la hermenéutica ontológica política o hermenéutica ontológica performativa, mediante la cual es posible comprender, interpretar, desocultar y promover transformaciones orientadas al agenciamiento (Alvarado et al., 2014; Luna et al., 2008). Dicho horizonte se desarrolló en el estudio a través de las narrativas colectivas y generativas, como relato de las experiencias vividas, método investigativo y posibilidad de creación. Las narrativas colectivas dan cuenta del carácter relacional, social y cultural presente en el contar los acontecimientos biográficos; las narrativas generativas vinculan las prácticas relacionales y dialógicas con las acciones y con el potencial del lenguaje para construir realidades, como propone Gergen (2007, 2012).

El grupo de participantes integró niños y niñas, entre 3 y 6 años de edad, sus familias - provenientes de contextos de conflicto armado-y sus docentes: en Bogotá participaron 16 niños, 8 niñas, 8 madres, 7 padres, 10 profesoras y 1 profesor del Centro de Desarrollo Infantil (CDI) Casita de los rincones; en Pereira participaron 11 niñas, 9 niños, 15 madres, 4 profesoras y la directora del CDI Re+Creo, perteneciente a la Corporación Crisol. Los niños y las niñas tenían diversas experiencias relacionadas con el conflicto armado: algunos y algunas habían vivido el desplazamiento forzado con sus familias a temprana edad, otros y otras lo habían vivido en etapa de gestación y, en otros casos, habían sido gestados después de que sus familias se encontraban viviendo en Bogotá o en Pereira. En cuanto a las familias, estas provenían de distintos departamentos como Caldas, Huila, Nariño, Cauca, Magdalena, Córdoba y Chocó, cuyos miembros participantes en el estudio tenían entre 22 y 50 años al momento del trabajo de campo y reportaban diversas vivencias en relación con el conflicto armado: el 66.6\% de las familias había vivido 
desplazamiento forzado; el 33.3\% había experimentado masacres, actos terroristas o atentados; el 33.3\% amenanzas; el 33.3\% desaparición forzada de un familiar; el 16.66\% reclutamiento por parte de un grupo armado y el 16.66 \% desmovilización de algún miembro. ${ }^{3}$ El grupo de agentes educativas y docentes tenía distintos niveles educativos: algunas entrenamiento técnico, otras estudios universitarios iniciados o finalizados y, la directora, maestría.

Para la selección de participantes se recurrió a la identificación de los lugares con mayor presencia de comunidades provenientes de contextos de conflicto armado en las ciudades. En Bogotá, el contacto se dio a través de la Secretaría Distrital de Integración Social, la cual estipuló que dicho CDI (ubicado en la localidad de San Cristóbal Sur) correspondía al que tenía mayor concentración de niños y niñas cuyas familias tenían carta de desplazamiento. En Pereira se contactó a la Corporación Crisol, entidad con una trayectoria significativa de trabajo con dicha población, buscando que los conocimientos y las transformaciones propiciados en el proceso tuvieran mayor perdurabilidad. En los dos CDI se convocó a todas las familias provenientes de contextos de conflicto armado, a sus hijos e hijas, así como al colectivo de docentes en interacción con ellos y ellas. Después de presentar la investigación a las instituciones y al grupo de participantes, se procedió a la firma de los consentimientos informados por parte de las y los docentes y de las familias de manera personal, así como a la solicitud de su autorización para la participación de los niños y las niñas. Para este mismo fin, con una obra de títeres, se presentó el estudio a los niños y las niñas, quienes además de informar verbalmente su interés en participar hicieron una seña, un dibujo o pusieron su nombre en el asentimiento informado.

La recolección de información y el agenciamiento se dieron mediante la implementación de quince talleres en cada CDI, doce de estos desarrollados con cada grupo de participantes por separado, cuatro con niños y niñas, cuatro con familias, cuatro con docentes y tres implementados con los tres grupos de actores reunidos en el mismo espacio. Los temas de los talleres fueron: presentación de la investigación y generación de lazos, subjetividades relacionales, externalización de las violencias, excepciones frente a las violencias, memorias, estrategias para el mantenimiento de los cambios y cierre ritual. En el taller de presentación cada participante declaró su interés por participar en el estudio: los adultos con el consentimiento informado, los niños y las niñas con el asentimiento

\footnotetext{
${ }^{3}$ La información acá presentada recoge las tendencias identificadas en la encuesta de caracterización del grupo de familias aplicada durante la investigación. Los porcentajes superan la totalidad (100\%) debido a que algunas familias experimentaron varias de las situaciones mencionadas.
} 
informado y del consentimiento por parte de sus acudientes. Entre las consideraciones éticas se tuvo presente la no revictimización del grupo de participantes y la garantía de confidencialidad y anonimato. Los talleres con los niños y las niñas tuvieron un énfasis lúdico y artístico, con la mediación de títeres, muñecos de plastilina, música, cuentos y juegos colectivos; con las familias y el grupo de docentes estos se enfocaron en contar historias, por medio de la elaboración de libros colectivos, una red tejida en lana, dramatizaciones, entre otras actividades. Todos los talleres se grabaron a través de audio y las narrativas emergentes en estos se transcribieron para el posterior análisis.

Así mismo, como parte del trabajo descrito, se analizaron las narrativas secundarias dilucidadas en la tesis doctoral Niños y niñas en contextos de conflicto armado: de la victimización a las narrativas que contribuyen a la construcción de paz, desarrollada por la primera autora de este artículo (Ospina-Alvarado, 2020a), con el acompañamiento de Kenneth Gergen, Gerrit Loots y Julia Villanueva, en un programa centrado en las bases del construccionismo social ofrecido por el Instituto Taos y la Universidad Libre de Bruselas.

Con las narrativas provenientes de los talleres y de la investigación en mención, se realizó un ejercicio de tematización, partiendo de la premisa de que el análisis temático de narrativas implica una doble interpretación: aquella desarrollada por los actores sociales frente a sus experiencias vitales y la desarrollada por quien investiga (Riessman, 2008). En este sentido, y como plantean Loots et al. (2013) y Tamboukou (2013), la tematización implica tejer sentidos y no fragmentarlos. En la investigación el tejido de significados logrado - consignado en los resultados- incorporó los relatos directos del grupo de participantes, las descripciones frente a estos y las interpretaciones de la investigadora puestos en diálogo con algunos referentes conceptuales. Emergieron en dicho ejercicio tres temas como hallazgos centrales, cada uno con sus subtemas: 1) la violencia como acontecimiento biográfico en la vida familiar; 2) potencias y posibilidades del resignificar: reconciliarse como seguir viviendo; y 3) la vida que merece ser vivida: salir adelante. El presente artículo se enfoca en el tercero de los hallazgos y sus subtemas emergentes: 3.1) salir adelante: una opción por las relaciones y los vínculos; 3.2) rompiendo con la homogeneización reproductora de la guerra: múltiples maneras de ser niños y niñas en relación; 3.3) prácticas de resistencia y re-existencia en los niños y las niñas; y 3.4) una vida que merece ser vivida: constitución de subjetividades e identidades políticas en el salir adelante (figura 1). 
Figura 1

Subtemas emergentes frente a La vida que merece ser vivida: salir adelante

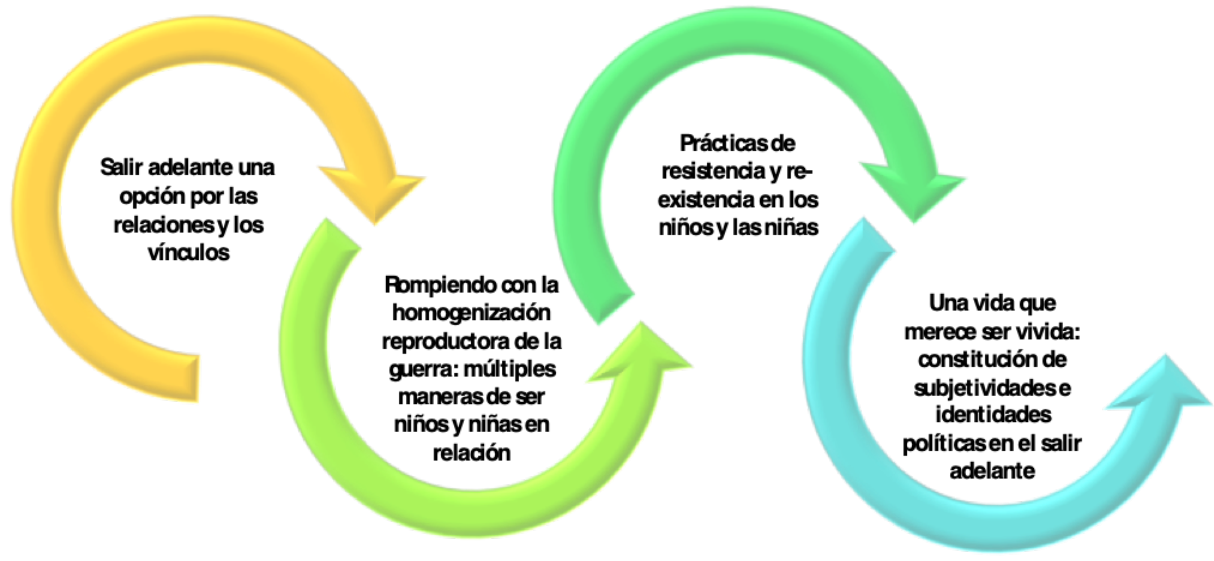

\section{Resultados}

Las subjetividades de los niños y las niñas, entendidas como los «sentidos propios que construye cada individuo sobre su ser y estar en el mundo (...), aquello que hace diferente a una persona de otra» (Alvarado et al., 2012, pp. 69-70), aparecieron en la presente investigación como vidas que merecen ser vividas por su humanidad y se vieron reflejadas en la metáfora emergente de las voces de los propios actores participantes: salir adelante. Esta metáfora implica una opción por las relaciones y los vínculos, romper con la homogeneización en la que se reproducen las violencias asociadas al conflicto armado, participar en prácticas de resistencia y re-existencia y aportar a la constitución de subjetividades e identidades políticas.

\section{Salir adelante: una opción por las relaciones y los vínculos}

Las vidas de los niños y las niñas de la primera infancia cuyas familias provienen de contextos de conflicto armado, al salir adelante se alejan de la reproducción de las violencias y de la ruptura de tejidos que experienciaron sus familias y se expresan en la importancia dada a las relaciones o al estar con los otros y las otras, que en momentos se presenta en tensión frente al abandono de algunos niños y algunas niñas por parte de su padre. También se expresan en prácticas relacionales de las familias como el diálogo, el ejemplo en la interacción y la importancia de la familia extensa, así como en las relaciones pedagógicas fundadas en el contar con, el disfrute, el afecto y el reconocimiento de las 
niñas y los niños en su humanidad. Finalmente, esto también se manifiesta en los aprendizajes que son mutuos y no solo se dan unidireccionalmente del mundo adulto al de los niños y las niñas por medio del ejemplo.

$\mathrm{Al}$ indagar a los niños y las niñas por aquello que más disfrutan de sus familias, estos refieren la importancia en sus vidas del estar con otros y otras; elemento que se opone a la reproducción pasiva frente a las violencias y a la ruptura de las relaciones presentes en los contextos de conflicto armado. La práctica relacional - o el estar con- se presenta de manera cotidiana en la vida de los niños y las niñas en escenarios como la casa familiar:

Investigadora: $\mathrm{Y}$ ¿qué es lo que más te gusta hacer con tu familia en la casa?

Niña 2b: Estar con mi mamá y con mi papá.

Cabe destacar que la apuesta de las niñas y los niños por las relaciones es relevante, en cuanto a que, como lo ha develado Uribe (1999), en los territorios marcados por el conflicto armado se generan ciertas tramas sociales en las que la violencia es vivida como natural. En este sentido, el estar con en los niños y las niñas da cuenta de aquello que Gergen (2012) ha argumentado como el potencial transformador presente en las relaciones y se enmarca en aquella responsabilidad por la vida de la otra persona que ha encontrado Mèlich (2000) en contextos como los del holocausto.

En tensión frente al valor de lo relacional, aparece también el abandono de algunos niños y algunas niñas por parte del padre, al que se suma el abandono que sienten sus madres y el vacío que emerge cuando el padre no permite que sus hijos o hijas sean registrados legalmente con su apellido:

Madre 2: Pues que quedé en embarazo y el papá no me respondió ni de la una ni de la otra, porque la mayor pues en el embarazo bien, pero pues cuando nació ya no y ya la segunda me la negó.

Como lo plantea esta madre en la expresión «me la negó», el valor relacional y, en este caso, la ausencia de relación genera una marca en la constitución de los niños y las niñas como sujetos. La experiencia de ser abandonado, negado o de ser madre soltera tiene implicaciones fuertes en la constitución subjetiva, en un país como Colombia en el que quien no tiene el apellido de sus padres pasa de ser reconocido a ser, como lo dirían Davies y Harré (1999), posicionado como hijo bastardo. La manera en la que son nombrados los niños y las niñas y cómo se nombran a sí mismos es de gran importancia, dado 
que existimos en el lenguaje (Shotter, 2008) y creamos nuestra existencia a partir de los sentidos compartidos (Gergen, 2012). El abandono posicionaría a estos niños y niñas en la vivencia de la precariedad, en el sentido de vidas que no merecen ser reconocidas; como propone Agamben (1998), nuda vida.

El estar con como opción por las relaciones y los vínculos se expresa en el diálogo del que participan los niños y las niñas como interlocutores válidos, el ejemplo que reciben de sus familias y docentes y la importancia que tiene para las familias la presencia de la familia extensa. El siguiente fragmento aborda algunos de estos elementos; en particular, destaca la relevancia del diálogo como práctica relacional y el ejemplo que enseña el posicionamiento ético tanto en la familia nuclear como en la familia extensa:

Madre 2: Que nosotros entre sí hablamos mucho; al menos cuando ellas están presentes procuramos pronunciar buenas palabras para que ellas nos entiendan y darles como un ejemplo (...). Y con el contacto físico, a veces le mostramos qué es bueno, qué es malo, qué es una caricia de amor y qué es una caricia de abuso. Eso yo les enseño mucho y mi familia también.

En cuanto a las relaciones pedagógicas, el estar con toma la forma de contar con la otra persona. Se refiere aquí a la disponibilidad por parte de los docentes frente a los niños y las niñas en prácticas relacionales mediadas por el disfrute, el afecto, el reconocimiento y la escucha frente a ellos y ellas, más allá de las vivencias de sus familias en los contextos de conflicto armado, a través de su humanidad:

Profesor 1: Yo creo que es disposición [la que debo tener para que sea realidad el jardín de los sueños]; disponer de todas las energías y de todas las habilidades en pro de los niños y la comunidad. Eso es como lo básico. Estar siempre disponible para cualquier inquietud, disponible para los mismos niños. Ellos también necesitan reír, saltar, jugar, brincar, no que los cohiban en un contexto diferente donde, no solo se aprendan letras y números, sino que también se aprenda la parte afectiva. Donde se les dé cariño, afecto, comprensión y donde se les lleve a un punto donde se eliminen los límites y se evalúe si los límites es ponerlos aquí o más arriba (...). Y en escucha porque ellos también tienen muchas cosas para contarle a uno; ellos no solamente le cuentan lo que quieren sino lo que se imaginan; entonces en algo podrá uno ayudarles.

El reconocimiento de los niños y las niñas, de sus voces, sus conocimientos, su imaginación y su creatividad, como sucede en el relato de este docente, y no exclusivamente en relación con lo que ellos, ellas o sus familias han vivido en contextos de conflicto armado 
da cuenta de aquello que White y Epston (1993) han denominado nombrar a las personas al margen del problema. Así mismo, el afecto, siguiendo a Manrique-Palacio et al. (2018), es una alternativa para construir paz.

La importancia dada a las relaciones - o al estar con- se expresó adicionalmente como hallazgos de la investigación en la identificación de los aprendizajes que emergen en las relaciones. Lo interesante es que los aprendizajes, tal y como refieren madres, padres y docentes son mutuos y no solamente ejercidos de manera lineal por medio de las personas adultas que enseñan a los niños y las niñas. Los aprendizajes emergen en el entramado relacional, de manera bidireccional, con el ejemplo que dan las distintas personas, niños, niñas, familias, docentes y comunidad:

Madre 4: Que por lo menos en el jardín las profesoras les enseñan cosas buenas a los niños $y$, a veces, los niños van y le dicen a uno lo que han visto o, a veces, hasta ellos le enseñan a uno. Por lo menos en los vecinos, mirar que de pronto comparten más en familia que uno, pues yo diría eso.

Es así como el estar con, como modo de resistencia a la reproducción de la violencia y como alternativa en pro de la construcción del mundo relacional, en el salir adelante se expresa en los distintos entornos relacionales en los que participan los niños y las niñas. Este modo de resistencia se da con múltiples matices y como su opción preferida de construcción de historia de vida y de apertura al mundo. Esto les posibilita apartarse de una individualidad única y abrirse a la multiplicidad que emerge en el entramado relacional.

\section{Rompiendo con la homogeneización reproductora de la} guerra: múltiples maneras de ser niños y niñas en relación

En los resultados del presente estudio es posible observar que no hay una única subjetividad o una única identidad — de los niños y las niñas de la primera infancia cuyas familias provienen de contextos de conflicto armado- ligada a su reproducción de la violencia, sino múltiples maneras de ser en las relaciones en las que participan; ello por medio de sus particularidades según el género o la edad y según sus vivencias y las de sus familias tanto en los contextos de conflicto armado como en la vida cotidiana.

En relación con las particularidades de género se encontró que hay configuraciones subjetivas que reproducen patrones culturales e históricos del modelo heteronormativo en las relaciones de género, semejante a la colonialidad cuestionada por Segato (2016), según los cuales los niños juegan a ser Transformers y las niñas a la cocinita. Por su parte, 
el grupo de docentes reconoce que los padres les enseñan a sus hijos la reproducción de la violencia, mientras que la enseñanza de las madres a las hijas se centra en valores. Siguiendo con esta tendencia, refieren una mayor participación de las madres en la institución educativa que de los padres, aun cuando aparecen también algunas contraprácticas que dan cuenta de ciertas rupturas como sucede, por ejemplo, con la referencia a que tanto las madres como los padres se vinculan al proceso educativo de sus hijos e hijas, a que ellas también trabajan y en unas ocasiones los padres participan en las actividades del jardín o a que las relaciones de género varían y no hay un patrón homogéneo:

Profesora 1: Pues es que también es tan variante...; tengo uno que es desplazado que es una familia muy bonita, a mi forma y mi concepción de ver, aunque el niño tiene muchas falencias. Pero son una familia digamos que unida y quieren cooperar y ayudar cuando se les dice algo sobre el niño. Y las otras que tengo como desplazadas las veo como familias en las que hay muchos conflictos entre las parejas, donde las más activas y más participativas en cuanto a la institución son las madres, y padres muy pasivos casi que ausentes del jardín, así lo describiría yo en mi caso con mis familias.

Profesora 5: En los míos es... también pues varía mucho; pero digamos en esos casos puntuales casi todos pues están pendientes de los niños, tanto papá como la mamá. La mayoría de ellas trabajan, creo, que como independientes o algo así. Entonces algunos tiempos pues se ajustan para poder estar en las actividades de los niños y las que se programan en el jardín se turnan, a veces vienen papás, a veces vienen mamás.

Adicionalmente al género, algunos aspectos puntuales emergieron a partir de la edad de los niños y las niñas. Esto implica que ellos y ellas, no solo sean leídos a través de la experiencia de sus familias en el contexto del conflicto armado, sino del estadio del ciclo vital en el que se encuentran. Sin embargo, este tipo de relatos también se ubican en aquello que Foucault (2009) y Gergen $(2006,2007)$ han denunciado como marcos normativos y discursos hegemónicos de normalidad. En el siguiente fragmento se refieren ciertas particularidades en cada niño o niña de la primera infancia cuyas familias provienen de contextos de conflicto armado, así como la normalidad de sus comportamientos de cara a su edad:

Profesora 5: Aquí en el jardín algunos son agresivos, otros son dispersos y otros son normales, o sea (...), tienen su comportamiento normal: (...) hay algunos que se caracterizan por ser agresivos, otros por ser muy pasivos, otros por agresivos-distraídos y otros por tener un comportamiento normal, más correspondiente a la edad en la que ellos están. 
A los aspectos descritos se suma la referencia a determinadas vivencias de los niños y las niñas o de sus familias en los contextos de conflicto armado, en el mismo sentido en que Uribe (1999) plantea que en dichos contextos hay ciertas experiencias comunes que marcan identidad y otras singulares, la referencia a las vivencias propias y aquellas de sus familias más allá del conflicto armado, que en momentos se enmarcan en otros contextos de violencia y vulnerabilidad. Como se muestra en el siguiente fragmento, dichas experiencias singulares son expresión de vivencias diferentes frente al desplazamiento forzado para cada niño o niña:

Profesora 2: No se puede generalizar y dar una característica determinada de los niños en condición de desplazamiento porque es muy difícil; o sea cada uno ha presentado la condición de desplazamiento diferente. Entonces, uno muchas veces hasta uno la desconoce.

Los relatos de las y los docentes y, en algunos casos, de los niños y las niñas, dan cuenta de las múltiples subjetividades e identidades que constituyen las vidas de estos niños y las niñas. Lo anterior muestra que ellos y ellas no se han constituido como masa homogénea reproductora de la violencia.

\section{Prácticas de resistencia y re-existencia en los niños y las niñas}

Entre las prácticas de resistencia frente a la reproducción de la violencia y de reexistencia como manera de existir alternativa frente a esta, emergieron en el presente estudio el cuidado que los niños y las niñas tienen de sí y de su cuerpo, sus prácticas relacionales éticas y su goce creativo.

El cuidado de sí y el cuidado del cuerpo apareció como parte de su vida cotidiana en prácticas como la alimentación, las acciones relacionadas con su salud y con su cuerpo, así como en valorar el alimento y el cuidado que reciben por parte de sus familias o docentes:

Investigadora: ¿Qué te gusta del jardín?

Niña 4: Comer.

Los relatos de niñas, niños y familias mostraron la vida y la salud como elementos centrales en el salir adelante como práctica de re-existencia. A la vez, apareció el discurso de la salubridad (Pedraza, 1999) y del cuidado del cuerpo como posibilidad de apertura al mundo y de encuentro con otros y otras. 
Un hallazgo importante fue la aparición en las niñas y los niños de expresiones éticas, a través de prácticas relacionales como la empatía, el respeto, la colaboración y la generosidad: «A él le gusta ser muy (...) servicial, compartir con el otro» (Madre 18). En este tipo de prácticas se hacen responsables de la vida de los otros y las otras (Levinas, 2006; Mèlich, 2000) y muestran el potencial presente en las relaciones (Gergen, 2007, 2009, 2012) al enmarcarse en una ética relacional (McNamee, 2015) y en los procesos colaborativos (Anderson, 2012).

Sin embargo, en algunos relatos de familias y docentes enmarcados en el deber ser aparecen también expresiones como «que sean niños de bien» (Madre 1), cuyos marcos normativos dan cuenta de lecturas angelizantes acerca de los niños y las niñas, como aquellas que denuncian Sosenski y Jackson (2012).

Otra disposición para la re-existencia en los niños y las niñas surgió en el goce creativo; la mayor parte de sus relatos dan cuenta de este al mencionar que lo que más les gusta es jugar, lo que más les gusta de sus amigos y amigas es jugar con ellos y ellas, implicándose a sí mismos y al entramado relacional en la descripción del otro. Aparece entonces el juego como posibilitador de la amistad y, al mismo tiempo, esta funge como posibilitadora de aquel en prácticas relacionales marcadas por la risa y el goce del encuentro con otros y otras:

Investigadora: ¿Por qué te gusta jugar con [niño 5]?

Niño 9: Porque es mi amigo.

Investigadora: $\mathrm{Y}$ ¿por qué es tu amigo?

Niño 9: Porque jugamos.

El goce y la risa que surgen en la amistad evocan aquella mención de Derrida (1998) acerca de la risa entre amigos, que implica un orden distinto al de la conversación, similar al orden de la imaginación descrito por Camps (2011).

De esta manera, a través de la vida cotidiana y de las relaciones en las que participan, los niños y las niñas logran resistirse a la reproducción de los círculos de la violencia y construyen disposiciones para la re-existencia. 


\section{Una vida que merece ser vivida: constitución de subje- tividades e identidades políticas en el salir adelante}

La investigación mostró que las vidas de los niños y las niñas merecen ser vividas: las vivencias de sus familias en contextos de conflicto armado no les quitan su condición de humanidad. El salir adelante en ellos y ellas da cuenta de la constitución de sus subjetividades e identidades políticas desde los primeros años, al construir unas vidas en las que no repiten la violencia que estuvo presente en las vidas de sus madres y padres y se orientan a la exploración, los interrogantes, la creatividad y las posibilidades que ofrece el futuro.

Por otra parte, en los relatos de familias y docentes salió a la luz el tema de la no repetición por parte de los niños y las niñas de la vida de sus padres y madres, no porque estas vidas no merecieran ser vividas, sino porque no querían que los niños y las niñas repitieran las historias de vulneración y violencia. Así, aportan al fortalecimiento de su autonomía relacional, su agenciamiento y su capacidad de afrontamiento, por medio de la metáfora del salir adelante:

Madre 4: Pues, si uno por decir vivió violencia, como uno no debe reflejar eso en los niños.

Madre 8: Sacarlos adelante, ¿no? Sacarlos adelante y que no vivan lo que uno vivió; sacarlos adelante cada día en esta ciudad tan grande.

Lo anterior muestra la posibilidad de romper con la tradición sin olvidar los aprendizajes de las vivencias pasadas (Gadamer, 1993), logrando el equilibro entre la materialidad del mundo y la condición humana (Arendt, 2009); y orientándose a los recursos y las potencias individuales y relacionales que se presentan también en contextos de violencia (Barudy \& Marquebreucq, 2006; Pérez-Sales, 2004).

Las disposiciones de los niños y las niñas para su constitución como sujetos políticos se identificaron mediante su exploración, el ser activos, el preguntarse por lo novedoso, como correlato del pensamiento crítico presente en el diálogo y en la creatividad, como expresiones subjetivas e identitarias en la primera infancia:

Profesor 1: Las estrategias de creatividad, pensemos siempre en que el niño explore esas ganas de preguntar, ese pensamiento crítico de decir: «yo quiero preguntar: ¿por qué sale el sol?, ¿por qué nacen los niños?, ¿para qué sirve tal cosa, tal otra?». Y eso nos ayudará también a estar más tranquilos a través del diálogo. 
Las preguntas de los niños y las niñas en sus primeros años frente al porqué de las cosas como disposición e interés por el mundo que les rodea remiten a la permanente pregunta por el sentido que ha planteado Heidegger (1951).

Por último, como disposición política expresada en el salir adelante como conexión entre el pasado y el futuro a través de acciones y relaciones presentes, surgió en los niños y las niñas su orientación a posibilidades futuras en el deseo de profesionalización: «[Cuando sea grande quiero ser] Doctor» (Niño g). Lo cual se conecta con la referencia de Santos (2011) a la orientación, las potencialidades y las posibilidades en lo que todavía no ha sucedido.

Es así como en la motivación por salir adelante que tienen los niños y las niñas, al igual que en el interés de sus familias por que salgan adelante, se gestan las bases y las disposiciones, desde los primeros años, para el encuentro con otros y otras, para abrirse al mundo y para buscar propiciar pequeñas transformaciones.

\section{Discusión}

Las principales contribuciones del estudio para dar respuesta a la pregunta acerca de cómo es el proceso de construcción relacional y social de subjetividades políticas de niños y niñas de la primera infancia en contextos de conflicto armado es la aparición de estos niños y niñas cuyas familias han vivido el destierro como vidas que merecen ser vividas por su condición de humanidad. Se trata de vidas singulares que no posibilitan hablar acerca de ellos, ellas o sus familias como un caso que responde a un patrón normativo único; vidas cuyas subjetividades políticas surgen en el salir adelante que da cuenta del potencial transformador de las relaciones en las que participan con sus familias, docentes, amigas y amigos; ello mediante prácticas relacionales, éticas como el respeto, la colaboración, la empatía, el cuidado de sí y de los demás, la confianza y la amistad, en las que, en encuentros intergeneracionales y con sus pares, construyen condiciones para la vida en común a través de su creatividad, su exploración del mundo y su indagación reflexiva.

Todo esto permite concluir que la subjetividad de los niños y las niñas cuyas familias provienen de contextos de conflicto armado no es homogénea. Existen experiencias relacionales y contextuales que han marcado diversas vivencias, incluso frente a acontecimientos comunes como el desplazamiento forzado. Así mismo, es en las relaciones en las que participan, como plantea Gergen (2012), donde se encuentra el mayor potencial de 
transformación y, en el caso del presente estudio, su constitución como sujetos políticos capaces de aprender de las experiencias y memorias de sus familias, pero de no reproducir las prácticas de violencia disponibles en los contextos de conflicto armado.

Las anteriores contribuciones se apartan de la tendencia presente en las investigaciones previas revisadas en Colombia (Bello \& Ruiz, 2002; Ceballos \& Bello, 2001; Defensoría del Pueblo, 2002; Human Rights Watch, 2003; Lozano, 2005; Niño, 2012; Sierra et al., 2009; Torrado et al., 2002; Torrado et al., 2009; Universidad Nacional de Colombia \& Observatorio sobre Infancia, 2002) y en otros países (Andersson, 2015; Bradley, 2018; Catani et al., 2010; Cummings et al., 2017; Demause, 2008; Feldman et al., 2010; Fossion et al., 2013; Haapanen et al., 2018; Jordans et al., 2016; Llabre \& Hadi, 2009; Llabre et al., 2015; Nagata, 1991; Peltonen \& Punamäki, 2010; Pye \& Simpson, 2017; Shenoda et al., 2018; Slone \& Mann, 2016; Sommer et al., 2018; Yurtbay et al., 2003; Zhang et al., 2018), las cuales se han centrado principalmente en la violencia y su reproducción, así como en la vulneración de los derechos de los niños y las niñas en los contextos de conflicto armado y guerra.

La centralidad de las relaciones en las que participan los niños y las niñas como potencial transformador frente a la violencia se acerca a los hallazgos de pocos estudios previos orientados a niños y niñas mayores (Alvarado et al., 2012; Ospina-Alvarado et al., 2018) y a jóvenes (Lugo, 2017), mas no a niños y niñas de la primera infancia cuyas familias han vivido el destierro. Cabe señalar que, como se encontró en el presente estudio, dicho potencial transformador requiere la toma de decisión y la participación creativa y activa, lo que se relaciona con el planteamiento de Camps (2011) de que se necesita transformar los afectos para ser dueño de sí y potenciar la acción; así como también con el de Derrida (1998) acerca de la acción que se requiere para desprenderse del destino o construirlo.

Las principales fortalezas del presente estudio son la emergencia de una metáfora como salir adelante, lo que en términos de Arendt (2009) da cuenta de la emergencia del pensamiento y, sumaríamos en esta investigación, posibilita la potenciación de los sujetos y el fortalecimiento de sus relaciones, así como el avance en el campo del conocimiento de la socialización política y del construccionismo social en términos de la construcción relacional y dialógica de subjetividades políticas en los primeros años de vida.

Los principales límites consistieron en no haber contado con algunos actores que sería importante contemplar en futuros estudios (como el grupo de pares); en este caso, los demás niños y niñas del CDI, el grupo de profesionales de la institución, más allá de las y los docentes y los agentes comunitarios. A futuro se podría también continuar for- 
taleciendo el campo de conocimiento de la subjetividad política de la primera infancia como práctica relacional y dialógica en otros contextos.

En los programas educativos de la primera infancia y en la política pública orientada a esta población será crucial trascender la mirada de su protección integral, reconociendo la importancia de las voces de los niños y las niñas desde sus primeros años, su lugar como sujetos sociales legítimos que no deben ser representados desde el mundo adulto sino escuchados; así como reconocer la centralidad de las relaciones en las que participan y sus disposiciones para la constitución de subjetividades políticas como construcción de futuro a través de acciones presentes que resignifican el pasado de sus familias en el contexto de la metáfora de salir adelante.

\section{Referencias}

Agamben, G. (1998). Homo sacer: el poder soberano y la nuda vida. Valencia: Pre-textos. Alvarado, S. V., Gómez, A., Ospina-Alvarado, M. C., \& Ospina, H. F. (2014). La hermenéutica ontológica política o hermenéutica performativa: una propuesta epistémica y metodológica. Revista Nómadas, (40), 207-220.

Alvarado, S. V., Ospina, H. F., Quintero, M., Luna, M. T., Ospina-Alvarado, M. C., \& Patiño, J. A. (2012). Las escuelas como territorios de paz. Construcción social del niño y la niña como sujetos políticos en contextos de conflicto armado. Universidad de Manizales; Cinde; Clacso.

Alvarado, S. V., \& Ospina-Alvarado, M. C. (2009). Contexto teórico para la reflexión sobre la socialización política. En G. Tonon (Comp.), Comunidad, participación y socialización política. Espacio Editorial.

Anderson, H. (2012). Collaborative relationships and dialogic conversations: Ideas for a relationally responsive practice. Family Process, 51(1), 8-24. https://doi.org/gxhs

Andersson, P. (2015). Quality of the relationship between origin of childhood perception of attachment and outcome of attachment associated with diagnosis of PTSD in adult Finnish war children and Finnish combat veterans from World War II (1939-1945) DSM-IV applications of the attachment theory. International Psychogeriatrics, $27(6)$, 1039-1048. https://doi.org/10.1017/S1041610215000101

Arendt, H. (2009). La condición humana. Paidós.

Banco Mundial. (2019). Indice de Gini. https://bit.ly/3CQRKcp 
Barudy, J., \& Marquebreucq, A. P. (2006). Hijas e hijos de madres resilientes. Traumas infantiles en situaciones extremas: violencia de género, guerra, genocidio, persecución y exilio. Gedisa.

Bello, M. N., \& Ruiz, S. (2002). Conflicto armado, niñez y juventud: una perspectiva psicosocial. Universidad Nacional de Colombia.

Boothby, N., Crawford, J., \& Halperin, J. (2006). Mozambique child soldier life outcome study: Lessons learned in rehabilitation and reintegration efforts. Global Public Health, 1(1), 87-107. https://doi.org/10.1080/17441690500324347

Bradley, S. (2018). Domestic and family violence in post-conflict communities: International human rights law and the state's obligation to protect women and children. Health and Human Rights Journal, 20(2), 123-136.

Camps, V. (2011). El gobierno de las emociones. Herder.

Castellanos, J., \& Torres, W. (2008). Una revisión de la producción académica sobre la violencia política en Colombia para indagar sobre el lugar de los jóvenes y las jóvenes. Revista Latinoamericana de Ciencias Sociales, Niñez y Juventud, 6(2), 523-563.

Catani, C., Gewirtz, A. H., Wieling, E., Schauer, E., Elbert, T., \& Neuner, F. (2010). Tsunami, war, and cumulative risk in the lives of Sri Lanka schoolchildren. Child Development, 81(4), 1176-1191. https://doi.org/10.1111/j.1467-8624.2010.01461.x

Ceballos, S., \& Bello, M. N. (2001). Conflicto armado, niñez y juventud: una perspectiva psicosocial. Universidad Nacional de Colombia.

Coalico. (2019). Boletín de monitoreo $N^{o}$. 21: Niñez y conflicto armado en Colombia, Edición especial. https://bit.ly/3CUuI4G

Comisión Histórica del Conflicto y sus Víctimas. (2015). Informe de la Comisión Histórica del Conflicto y sus Víctimas: contribución al entendimiento del conflicto armado en Colombia. Mesa de Conversaciones.

Contreras, M. H. (2003). El conflicto armado en Colombia. Revista de Derecho, 19, 119-125.

Cummings, E. M., Merrilees, C. E., Taylor, L. K., \& Mondi, C. F. (2017). Developmental and social-ecological perspectives on children, political violence, and armed conflict. Development and Psychopathology, 29(1), 1-10. https://doi.org/ggjrsq

Davies, B., \& Harré, R. (1999). Posicionamiento: la producción discursiva de la identidad. Sociológica, 14(39), 215-239.

Defensoría del Pueblo. (2002). La niñez en el conflicto armado colombiano. Unicef Colombia, Defensoría del Pueblo. Boletín Niñez n. ํ 8. https://bit.ly/3CQM7Lh

Demause, L. (2008). The childhood origins of World War II and the Holocaust. Journal of Psychohistory, 36(1), 2-30. Pmid: 19043997

Derrida, J. (1998). La amistad como alianza en el silencio: políticas de la amistad. Trotta. 
Feldman, M., Taïeb, O., \& Moro, M. R. (2010). Jewish children hidden in France between 1940 and 1944: An analysis of their narratives today. American Journal of Orthopsychiatry, 8o(4), 547-556. https://doi.org/10.1111/j.1939-0025.2010.01059.x

Fossion, P., Leys, C., Kempenaers, C., Braun, S., Verbanck, P., \& Linkowski, P. (2013). Depression, anxiety and loss of resilience after multiple traumas: An illustration of a mediated moderation model of sensitization in a group of children who survived the nazi holocaust. Journal of Affective Disorders, 151(3), 973-979. https://doi.org/f5fsxq Foucault, M. (2009). Vigilar y castigar: nacimiento de la prisión. Siglo XXI.

Gadamer, H. (1993). Verdad y método. Sígueme.

Garay, L. J., Salcedo-Albarán, E., De León-Beltrán, I., \& Guerrero, B. (2008). La captura y reconfiguración cooptada del Estado en Colombia. Fundación Método; Fundación Avina; Transparencia por Colombia.

Gaviria, M. B. (2012). Pluralidad humana en el destierro: tejido de la memoria singular de cuerpos vividos en el destierro en Colombia [Tesis doctoral, Cinde-Universidad de Manizales] Repositorio Cinde http://repository.cinde.org.co/handle/20.500.11907/502 Gergen, K. (1996). Realidades y relaciones: aproximaciones a la construcción social. Paidós. Gergen, K. (2006). El yo saturado: dilemas de identidad en el mundo. Paidós.

Gergen, K. (2007). Construccionismo social, aportes para el debate y la práctica. Universidad de los Andes.

Gergen, K. (2009). Relational being. Oxford University Press.

Gergen, K. (2012). Principios orientadores del construccionismo social. https://bit.ly/3ul3gdg

Gergen, M., \& Gergen, K. (2012). Playing with purpose: Adventures in performative social science. Left Coast Press.

González, F., Bolívar, I., \& Vásquez, T. (2002). Violencia política en Colombia: de la nación fragmentada a la construcción del Estado. Centro de Investigación y Educación Popular. Haapanen, M. J., Perälä, M. M., Salonen, M. K., Kajantie, E., Simonen, M., Pohjolainen, P., Pesonen, A. K., Räikkönen, K., Eriksson, J. G., \& Von Bonsdorff, M. B. (2018). Early life stress and frailty in old age: The Helsinki birth cohort study. BMC Geriatrics, 18(179), 1-8. https://doi.org/10.1186/s12877-018-0873-5

Heidegger, M. (1951). Construir, habitar, pensar. Universidad Católica de Chile.

Human Rights Watch. (2003). Aprenderás a no llorar: niños combatientes en Colombia. Gente Nueva.

Ila, P., Martínez, A., Arias, A., Núñez, P., \& Caicedo, M. (2009). Conflicto armado en la primera infancia en tres territorios colombianos: Putumayo, Magdalena Medio y 
Arauca. En A. Mejía (Ed.), Colombia: huellas del conflicto en la primera infancia (pp. 147-156). Número Ediciones.

Jordans, M. J., Pigott, H., \& Tol, W. A. (2016). Interventions for children affected by armed conflict: A systematic review of mental health and psychosocial support in low-and middle-income countries. Current Psychiatry Reports, 18(1), 1-15. https://doi.org//77876t

Levinas, E. (2006). Humanismo del otro hombre. Siglo XXI.

Llabre, M. M., Hadi, F., La Greca, A. M., \& Lai, B. S. (2015). Psychological distress in young adults exposed to war-related trauma in childhood. Journal of Clinical Child and Adolescent Psychology, 44(1), 169-18o. https://doi.org/10.1080/15374416.2013.828295

Llabre, M. M., \& Hadi, F. (2009). War-related exposure and psychological distress as predictors of health and sleep: A longitudinal study of Kuwaiti children. Psychosomatic Medicine, 71(7), 776-783. https://doi.org/10.1097/PSY.obo13e3181ae6aee

Loots, G., Coppens, K., \& Sermijn, J. (2013). Practising a rhizomatic perspective in narrative research. En M. Andrews, C. Squire, \& M. Tamboukou (Eds.), Doing narrative research. Sage.

Lozano, P. (2005). La guerra no es un juego de niños: historias de una infancia quebrada por el conflicto. Intermedio.

Lugo, V. (2017). Disarmed warriors: Narratives with youth ex-combatants in Colombia. Taos Institute Publications; WorldShare Books.

Luna, M. T. (2018). Cuerpo, territorio y política: una experiencia de construcción de paz. En M. T. Luna (Ed.), Segundo orden narrativo: lecciones aprendidas (pp. 67-106). Universidad Pedagógica Nacional; Universidad de Manizales; Cinde.

Luna, M. T., Botero, P., \& Alvarado, S. V. (2008). La comprensión y acontecimientos políticos, ¿es cuestión de método? Un aporte a las ciencias sociales. Revista Latinoamericana de Ciencias Sociales, Niñez y Juventud, 6(2), 52-75.

Manrique-Palacio, K. P., Zinke, L., \& Russo, A. R. (2018). Pisotón: un programa de desarrollo psicoafectivo como alternativa para construir la paz. Revista Latinoamericana de Ciencias Sociales, Niñez y Juventud, 16(1), 131-148. https://doi.org/gxhz

McNamee, S. (2015). Ethics as discursive potential. Australian and New Zealand Journal of Family Therapy, 36(4), 419-433. https://doi.org/10.1002/anzf.1125

Mèlich, J. C. (2000). Narración y hospitalidad: la ausencia del testimonio. Anthropos.

Nagata, D. K. (1991). Transgenerational impact of the japanese-american internment: Clinical issues in working with children of former internees. Psychotherapy, 28(1), 121-128. https://doi.org/10.1037/0033-3204.28.1.121 
Niño, N. (2012). Los derechos están torcidos: una aproximación desde el enfoque de género y generacional a la vivencia de los derechos de las niñas en zonas de conflicto (Cúcuta y Tibú). Fundación Creciendo Unidos. https://bit.ly/zodsclN

Ospina-Alvarado, M. C. (Ed.). (2018). Construcción social de niños y niñas: familias, docentes y otros agentes relacionales. Universidad Pedagógica Nacional; Universidad de Manizales; Cinde.

Ospina-Alvarado, M. C. (2020a). Niños y niñas en contexto de conflicto armado: de la victimización a narrativas que contribuyen a la construcción de paz [Tesis doctoral]. Free University of Brussels, Taos Institute.

Ospina-Alvarado, M. C. (2020b). Salir adelante: construcción relacional de subjetividades políticas de niños y niñas de la primera infancia cuyas familias provienen de contextos de conflicto armado [Tesis doctoral]. Universidad de Manizales-Cinde.

Ospina-Alvarado, M. C., Alvarado, S. V., Carmona, J. A., \& Arroyo, A. (Comps.) (2018). Construcción social de niñas y niños en contextos de conflicto armado: narrativas generativas para la construcción de paz. Universidad Pedagógica Nacional; Cinde; Universidad de Manizales.

Panter-Brick, C., Grimon, M., Kalin, M., \& Eggerman, M. (2015). Trauma memories, mental health, and resilience: A prospective study of afghan youth. Journal of Child Psychology and Psychiatry, and Allied Disciplines, 56(7), 814-825. https://doi.org/ggvpvh

Pedraza, Z. (1999). En cuerpo y alma: visiones del progreso y de la felicidad. Universidad de los Andes.

Peltonen, K., \& Punamäki, R. (2010). Preventive interventions among children exposed to trauma of armed conflict: A literature review. Aggressive Behavior, 36(2), 95-116. https://doi.org/10.1002/ab.20334

Pérez-Sales, P. (2004). El concepto de trauma y de respuesta al trauma en psicoterapia. Norte de Salud Mental, (20), 29-36.

Pye, R. E., \& Simpson, L. K. (2017). Family functioning differences across the deployment cycle in British army families: The perceptions of wives and children. Military Medicine, 182(9-10), e1856-e1863. https://doi.org/10.7205/milmed-d-16-00317

Richter, L. M., Lye, S. J., \& Proulx, K. (2018). Nurturing care for young children under conditions of fragility and conflict. En J. F. Leckman, \& P. R. Britto (Eds.), Towards a more peaceful world: The promise of early child development programmes: New directions for child and adolescent development (pp. 13-26). https://doi.org/10.1002/cad.20232

Riessman, C. K. (2008). Narrative methods for the human sciences. Sage. 
Romero, T., \& Castañeda, E. (2009). El conflicto armado colombiano y la primera infancia. En A. Mejía (Ed.), Colombia: huellas del conflicto en la primera infancia (pp. 31-53). Número Ediciones.

Santos, B. de S. (2011). Epistemologías del sur. Utopía y Praxis Latinoamericana, 16(54), 17-39. Save the Children. (2018). Análisis del rol de la niñez en los acuerdos de paz. Autor.

Segato, R. L. (2016). La norma y el sexo: frente estatal, patriarcado, desposesión, colonialidad. En K. A. Bidaseca (Coord.), Genealogías críticas de la colonialidad en América Latina, África, Oriente (pp. 31-64). Clacso; Idaes.

Shenoda, S., Kadir, A., Pitterman, S., \& Goldhagen, J. (2018). The effects of armed conflict on children. Pediatrics, 142 (6), 1-9, e20182585. https://doi.org/10.1542/peds.2018-2585

Shotter, J. (2008). Conversational realities revisited: Life, language, body and world. Taos Institute Publication.

Sierra, A., Lozano, P., Guerrero, A., \& Salamanca, N. (2009). Niños vinculados al conflicto, cubrimiento periodístico responsable. Medios para la Paz-MPP; Unión Europea; Fundación Colombia Multicolor.

Slone, M., \& Mann, S. (2016). Effects of war, terrorism and armed conflict on young children: A systematic review. Child Psychiatry \& Human Development, 47(6), 950-965. https://doi.org/10.1007/s10578-016-0626-7

Sommer, M., Munoz-Laboy, M., Wilkinson-Salamea, E., Arp, J., Falb, K., Rudahindwa, N., \& Stark, L. (2018). How narratives of fear shape girls' participation in community life in two conflict-affected populations. Violence Against Women, 24(5), 565-585. https://doi.org/10.1177/1077801217710000

Sosenski, S., \& Jackson, E. (2012). Introducción. Nuevas miradas a la historia de la infancia en América Latina: entre prácticas y representaciones. Universidad Nacional Autónoma de México.

Tamboukou, M. (2013). A Foucauldian approach to narratives. En M. Andrews, C. Squire, \& M. Tamboukou (Eds.), Doing narrative research. Sage Publications.

Torrado, M. C., Camargo, M., Pineda, N., \& Bejarano, D. (2009). Estado del arte sobre primera infancia en el conflicto. En A. Mejía (Ed.), Colombia: huellas del conflicto en la primera infancia (pp. 31-53). Número Ediciones.

Torrado, M. C., Durán, E., Serrato, L., Del Castillo, M., Buitrago, L. \& Acero, G. A. (2002). Niños, niñas y conflicto armado en Colombia: una aproximación al estado del arte 19902001. Convenio del Buen Trato.

Unidad para la Atención y Reparación Integral a las Víctimas. (2020). Registro único de víctimas. https://bit.ly/zase 815 
Universidad Nacional de Colombia \& Observatorio sobre Infancia. (2002). Niños, niñas y conflicto armado en Colombia: una aproximación al estado del arte, 1990-2001. Convenio del Buen Trato; Fundación Antonio Restrepo Barco.

Uribe, M. T. (1999). Las soberanías en disputa: ¿conflicto de identidades o de derechos? Estudios Políticos, (15), 23-45.

Villanueva O'Driscoll, J. (2013). If you prepare the garden, the plants will take care of themselves: insertion processes of children disengaged from armed groups in Colombia [Tesis de doctorado]. Vrije Universiteit Brussel; Universidad Católica Boliviana San Pablo.

Villanueva, J., Loots, G., Losantos, M., Exeni, S., Berckmans, I., \& Derluyn, I. (2017). Reinsertion processes of children disengaged from armed groups in Colombia: What is the problem represented to be? Revista Eleuthera, 16, 85-100.

White, M., \& Epston, D. (1993). Medios narrativos para fines terapéuticos. Paidós.

Yurtbay, T., Alyanak, B., Abali, O., Kaynak, N., \& Durukan, M. (2003). The psychological effects of forced emigration on muslim albanian children and adolescents. Community Mental Health Journal, 39(3), 203-212. https://doi.org/1023386122344

Zhang, N., Zhang, J., Gewirtz, A. H., \& Piehler, T. F. (2018). Improving parental emotion socialization in military families: Results of a randomized controlled trial. Journal of Family Psychology, 32(8), 1046-1056. https://doi.org/10.1037/famoooo461 